\title{
RESEARCH ARTICLE \\ Bitcoin Market Volatility Analysis Using Grand Canonical Minority Game
}

\author{
Matteo Ortisi ${ }^{*}$
}

\begin{abstract}
In this paper we propose to use the Grand Canonical Minority Game (GCMG, a highly simplified financial market model) as a model of bitcoin market to show how the lack of an income for "miners", similar to yield earned by bond holders, could be a structural reason for high volatility of bitcoin price in a reference currency. Coherently with present analysis, the introduction of future contracts on bitcoin would have the effect of reducing the overall market volatility.
\end{abstract}

\section{Introduction}

Starting from end of July 2010 bitcoin started to be exchanged versus US dollars and now it is easily possible to exchange bitcoins vs major currencies. ${ }^{1}$ Observing the bitcoin price time series (here we will consider the the price of 1 bitcoin in US Dollar), the most evident characteristic is its high volatility. Even discarding the "wild" years, culminating with the 2013 peak and subsequent fall at around the same time as the bankruptcy of MtGox exchange, price returns volatility is so high that its minimum can be compared with the level of SP500 Index volatility during the Lehman crisis, the most severe global financial crisis after second World War (see Figure 1).

Looking at the chart comparing bitcoin relative to USD price and volatility time series, we can approximately identify four sub-periods. The first one, ending at the beginning of 2013, is characterized by a constantly decreasing volatility. It coincides with the period when the price of bitcoin in USD slowly increased from the early days fraction of dollar to a quote around 30 USD. The second period, from the beginning of 2013 to the end of 2013, is characterized by a volatility sharp increase. In the same period bitcoin price soared to its historical maximum (around 1100 USD) reached just before the collapse of MtGox exchange in late 2013. The third period, ending at the beginning of 2015, shows a drop of the volatility that seems to be mainly due to more stable prices replacing most volatile ones in the observations window: the volatility increase toward its peak has been driven by upward movements of the price, whilst the subsequent price decrease has been relatively smoother. The last period, starting at beginning of 2015, is characterized by a more stable volatility ranging from $40 \%$ to $60 \%$, reflecting the relatively more stable bitcoin price of recent years. Here the volatility trend is the opposite of the price one: a decrease in volatility corresponds to an increase in price, reflecting a behavior similar to the one usually observed in the stock or commodities market. Despite that, volatility is still very high and it is

*M. Ortisi (matteo.ortisi@gmail.com) is a Senior Portfolio Construction Analyst at Pioneer Investments in Milan, Italy

${ }^{\dagger}$ 1Cr9PVH9dFz1KMxp3eMv6QN2MjPJWgVfQ9 

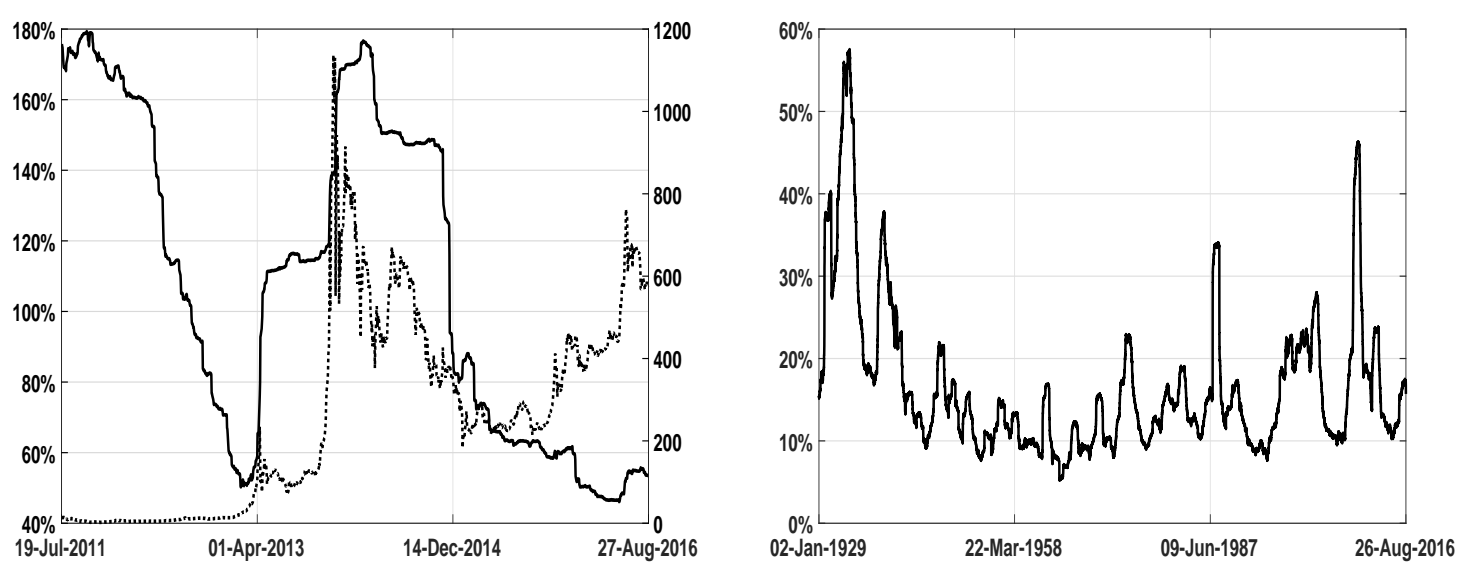

Fig. 1. Left: 1 year rolling volatility of bitcoin relative to USD daily returns vs date (continuous line and left axis) and daily price of bitcoin in USD (dotted line and right axes). Right: 1 year rolling volatility of SP500 Index daily returns vs date.

difficult for investors to approach bitcoin as a long term investment rather than a pure speculative one. It worth to note that, being the volatility of USD relative to other currencies, like EUR or CNY, a small fraction of bitcoin relative to USD one, the choice of the base currency is irrelevant in our analysis. Throughout the rest of the paper we will refer to USD as reference currency, without loss of generality.

Here we are proposing to use an interacting agents model, known as Grand Canonical Minority Game (GCMG), to better understand what could be a structural source of volatility. ${ }^{2}$ It is important to note that we are taking the point of view of an investor arguing about the possibility of investing in bitcoin as an asset class and not about the convenience of mining or not mining. As we will see later this is relevant in the definition of each agent action.

Under the name Minority Games lives a family of highly simplified toy models that in some variants have been shown to reproduce quite accurately the observable quantities of financial markets, like volatility and volume clustering, market crashes, fat tailed returns. ${ }^{3}$ The Minority Game has been introduced as a model to explain the complex dynamic underlying the El Farol Bar Problem. ${ }^{4}$ Among its several modification, the GCMG has been proved to be a good model of financial markets. ${ }^{3}$ Key ingredients of this model are the two types of traders that interplay: producers and speculators. Producers are traders that use the market to exchange goods; their trading decisions are driven by fundamental analysis of the economic activity and not from the price dynamics. They drive the underlying economic activity and in reaction to a news arrival process inject information into the market enrich the overall knowledge.

Speculators are the second type of traders. The first difference with respect to producers is the possibility of not trading if the market does not contain sufficiently profitable opportunities. They try to gain from market fluctuations; studying the relationship between news arrivals and market reactions, they aim to anticipate market movements. Similarly to the role of speculators (see Black), on the one hand they provide liquidity to the market and for producers, on the other hand they are responsible for observable quantities like market crashes, volatility and fat tailed returns. ${ }^{5}$ 
The market ecology just described suits not only classic financial markets, but bitcoin market too. The role of producers is played by a "core" group of miners that literally "mines" new bitcoins and validate transactions, and by long-term bitcoin holders. This kind of agent is driven by a fundamental belief in bitcoin and a long term view in the underlying system, so it is not interested in speculating on short term price fluctuations. Their investment strategies tend to be predictable: they are essentially buying (either directly or indirectly) or holding bitcoins in exchange of USD.

The role of speculator is played by both "small" miners and classic financial speculators. The former ones need to try to earn from price movements in order to compensate for the small chance they have to mine enough bitcoins to cover expenses; the latter ones speculate on bitcoin like they would do on any other currency pair, equity or commodity. Both of them have trading strategies aimed to take advantage of market fluctuations and can withdraw from the market if it is not profitable. Before coming again on these aspects and seeing how GCMG can help in understanding why the lack of an income in USD paying miners for their activity could be a relevant reason for high bitcoin price volatility, it worth to spend few words introducing GCMG and its capability to reproduce financial markets observable quantities.

\section{Introduction to GCMG Model and Dynamics}

For a complete introduction to Minority Games we refer to Moro. ${ }^{6}$ For a more detailed study of GCMG to Challet and Marsili.., 3

The Minority Game is a simple model based on Arthur's "El Farol" bar problem which describes the behavior of a group of competing heterogeneous agents subject to the economic law of supply and demand. ${ }^{4}$ In its original formulation the problem is as follows: a number of agents must decide independently each week whether to go to their favorite bar or not. If an agent expects that the bar will be overcrowded, he will stay at home; otherwise, he will go to the bar. If all agents believe few will go, then all will go, destroying all the expectations. On the other hand, if all agents believe many will go, then nobody will go, destroying the expectations again.

In its mathematical formulation, the Minority Game, $N$ agents have to decide in advance whether to go or not to go to the bar (to buy or to sell) and being in the minority group is the winning side: if most of the agents decide to go to the bar, it will be overcrowded and the choice of staying at home made by the minority group is the winning one; if most of the agents decide to stay at home, the opposite is true.

The model can be applied to the stock market: at time $t_{0}$ each agent has to put a buy or sell order to be executed at the subsequent instant $t_{1}$ : if the majority of the traders decided to buy (to sell), the price will increase (decrease) and the minority of them that put a sell (buy) order will sell (buy) at a higher (lower) price making a profit.

In the standard Minority Game all agents have to play at each time step, even if all their strategies are unprofitable; a natural extension is the Grand Canonical Minority Game where, if for some of them trading is unprofitable, they withdraw from the market. This characteristic makes it similar to what in physics is known as a Grand Canonical Ensemble (a system that can exchange particles with a reservoir).

Let us translate the above description into mathematical terms. In the Minority Game agents $i=1,2, \ldots, N$ submit a bid $b_{i}(t)$ to the market at the same instant $t=1,2, \ldots$. The winning side 
LEDGER VOL 1 (2016) 111-118

\begin{tabular}{|c|c|}
\hline Input & Output \\
\hline$-1-1-1$ & -1 \\
\hline$-1-1+1$ & -1 \\
\hline$-1+1-1$ & +1 \\
\hline$-1+1+1$ & -1 \\
\hline$+1-1-1$ & -1 \\
\hline$+1-1+1$ & +1 \\
\hline$+1+1-1$ & -1 \\
\hline$+1+1+1$ & +1 \\
\hline
\end{tabular}

Table 1. Example of a strategy with $m=3$.

is made of agents whose bid as the opposite sign of the total bid $A(t)=\sum_{i} b_{i}(t)$ : the minority side is the winning side. If the overall bid is positive (buy) the stock price will increase and the minority agents that submitted a negative bid (sell) will benefit from selling at a higher price. We assume that the agents have limited analyzing capability and they can only retain the last $m$ game results. A trading decision is hence based on the last $m$ outputs. A strategy is a mapping from the sequence of the last $m$ winning outcomes to the action of the agent. An example is given in Table 1.

Since there are $P=2^{m}$ possible inputs for each strategy, the total number of strategies is $2^{P}$. The bids of agents depend on the value $\mu(t)$ of public information available (one of the possible $P$ strategy inputs). Considering the binary representation of $\mu(t)$ (with -1 as 0 and +1 as 1 ), the public information can be seen as drawn from uniformly distributed integers $1, \ldots, P$. Each agent is assigned a trading strategy, randomly chosen from the set of $2^{P}$ possible strategies, that prescribes a bid $a_{i}^{\mu}= \pm 1$ for each information $\mu$. An agent bid has the form $b_{i}(t)=\phi_{i}(t) a_{i}^{\mu(t)}$ where $\phi_{i}(t)=1$ or 0 according to whether agent $i$ trades or not.

In order to assess the goodness of a strategy, and hence decide to trade or to refrain, at each strategy $i$ is assigned a score $U_{i}(t)$ that is updated on the basis of the overall outcome $A(t)=\sum_{i=1}^{N} b_{i}(t)=\frac{\sum_{i=1}^{N} \phi_{i}(t) a_{i}^{\mu(t)}}{N}$. A strategy score is updated recursively:

$$
U_{i}(t+1)=U_{i}(t)-a_{i}^{\mu(t)} A(t)-\varepsilon_{i} .
$$

Being $A(t)$ the overall bid $(A(t)>0$ if the majority bid is a buy, $A(t)<0$ if the majority bid is a sell), if the agent bid $a_{i}^{\mu(t)}$ falls in the minority side and $-a_{i}^{\mu(t)} A(t)$ is larger than $\varepsilon_{i}$, the score $U_{i}$ increases. The larger $U_{i}$ the more likely it is that the agent trades $\left(\operatorname{Prob}\left\{\phi_{i}(t)=1\right\}=\frac{1}{1+\exp ^{-U_{i}(t)}}\right)$, see Challet. ${ }^{7}$

The threshold $\varepsilon_{i}$ models the incentives of agents for trading in the market. A prudent investor is characterized by an $\varepsilon>0$ (he takes part to the market only if the expected return is higher than a risk free rate), whilst a speculator has $\varepsilon \leq 0$ (profit opportunities offered by the market are so high that he is available to pay in order to take part to the market). Agents that want to stay in the market no matter what because they have a fundamental view are modeled with $\varepsilon=-\infty$. The purpose of setting $\varepsilon=-\infty$ is to have the right hand term of Equation (1) equal to 
$+\infty: \operatorname{Prob}\left\{\phi_{i}(t)=1\right\}=1$ and the agent always plays its strategy. This is the case of miners that mine no matter what and long term investors, that have the strategy of being long bitcoin.

Being interested in understanding if the model just described could be a good candidate to simulate bitcoin market, we briefly outline the outcome of extensive simulations carried on GCMG (reported in Marsili), showing its capability of reproducing observable quantities common to all financial markets. ${ }^{3}$ We will come back on the role played by producers and speculators on the overall predictability and volatility later when discussing the bitcoin case.

Despite its simplicity, the model is able to reproduce complex phenomena like market crashes, volatility and volume clustering and fat tailed returns. ${ }^{3}$ The salient characteristics arising from performed simulations is that all the mentioned observable quantities depend on the number of speculators and the size of $\varepsilon$ : a large number of speculators rushing into the markets causes violent shocks followed by sudden crashes whose frequency increases with the number of speculators and decreases with $\varepsilon$; volatility clustering increases and returns tails become fatter and fatter as the number of speculators increases. All these conclusions hold of course in a simplified market: real markets are much more complicated having agents that trades on different shares using different instruments. But the bitcoin market is a new born market, where agents are trading only the currency pair. From this point of view it is more similar to a toy market than any other market. That is why we think that GCMG could be a pretty good model of it.

\section{Bitcoin Market Dynamics Explained Using Grand Canonical Minority Game}

The unique characteristics of bitcoin with respect to any other commodity is the way it is created. Since inception, the total amount of bitcoins that will be created through what is popularly known as mining process has an asymptotic limit of 21 million tokens; every four years the number of bitcoins created is scheduled to be cut in half until 2040 when creation is supposed to go to zero. Adding new transaction records to the blockchain (Bitcoin public ledger) involves a proof of work that becomes harder and harder and this activity at the moment is rewarded with new bitcoins and a fee in bitcoin only. This means that miners are structurally exposed to the fluctuations of bitcoin price in USD (they earn bitcoins and spend USD for mining resources).

Going back to the concepts we reported in previous section, an agent acting on bitcoin market is characterized by updating strategy score $U_{i}(t)$ as in Equation (1) with $\varepsilon_{i} \leq 0$ : agents not involved in mining activity have $\varepsilon_{i}=0$ (speculators that simply aim to earn from price fluctuations), agents involved in mining activity that needs to trade at least to cover mining cost have $-\infty<\varepsilon_{i}<0$, agents that mine no matter what and long-term bitcoin holders not exploiting price fluctuations have $\varepsilon_{i}=-\infty$.

Having all agents with $\varepsilon_{i} \leq 0$ is what makes bitcoin market (from a multi-agent game perspective) different from other financial markets where producers, whose decision are driven by fundamental views, have $\varepsilon_{i}=-\infty$, but there are risk-averse investors, with $\varepsilon_{i}>0$, who trade for profiting from price movements. The difference is due to the fact that in a classic financial market a speculator makes a decision on a cost-opportunity basis, comparing the potential gain from a trading decision with some minimum threshold, usually the risk free interest rate $\left(\varepsilon_{i}>0\right)$, whilst in bitcoin market there is not such a possibility and that threshold is the cost for being in the market ( $\varepsilon_{i}=0$ for not mining speculators, $\varepsilon_{i}<0$ for mining ones). 
Coherently with the model we just presented, "buy and hold" - like investors (buying directly bitcoin in exchange of USD or indirectly through the mining process) that are driven by fundamental, long term views are producers, whilst all other agents, both miners and not, are speculators. As we will see in next section, this market structure has a big impact on overall market volatility.

At this point it is important to introduce some relevant statistical measures. Two fundamental quantities of GCMG are predictability and volatility. They are defined, respectively, as

$$
H=\frac{1}{P} \sum_{\mu=1}^{P}\langle A \mid \mu\rangle^{2}
$$

and

$$
\sigma^{2}=\frac{1}{P} \sum_{\mu=1}^{P}\left\langle A^{2} \mid \mu\right\rangle
$$

where $\langle\cdot \mid \cdot\rangle$ denotes the conditional time average.

If $H_{0}>0$ the market is statistically predictable and arbitrage opportunities arise; if $H_{0}=0$ the market is efficient: arbitrage opportunities are exploited and hence disappear. Other fundamental quantities are the reduced numbers of speculators, producers and active speculators defined respectively as:

$$
n_{s}=\frac{N_{s}}{P}, \quad n_{p}=\frac{N_{p}}{P}, \quad n_{a c t}=\frac{\sum_{i}\left\langle\phi_{i}(t)\right\rangle}{P},
$$

where $N_{s}$ and $N_{p}$ are the total number of speculators and producers.

Following Challet, ${ }^{7}$ we define the price dynamics in terms of the excess demand as

$$
\log p(t+1)=\log p(t)+A(t) .
$$

Market volatility is defined as the square root of price returns variance and by Equation (2)

$$
v_{m k t}=\sqrt{\operatorname{Var}\left[\log \frac{p(t+1)}{p(t)}\right]}=\sqrt{\operatorname{Var}[A(t)]}=\sqrt{\frac{1}{P} \sum_{\mu=1}^{P}\left\langle A^{2} \mid \mu\right\rangle}=\sigma .
$$

The role played by parameters $\varepsilon, n_{s}$ and $n_{a c t}$ in the volatility dynamics is clearly explained by Figure 2. The upper part shows the behavior of the average number of active traders $\left\langle n_{a c t}\right\rangle$ (vertical axis) as a function of reduced number of speculators $n_{s}$ (horizontal axis), both for $\varepsilon>0$ (open symbols) and $\varepsilon<0$ (full symbols). For $n_{s}<1$ the two curves roughly overlap, whilst they drastically diverge as $n_{s}$ increases.

The lower part shows $\frac{\sigma^{2}}{P}$ and $\frac{H}{P}$ as a function of $n_{s}$. The highest of the two curves is the volatility $\frac{\sigma^{2}}{P}$. For relatively low value of the reduced number of speculators $n_{s}$ its behavior for $\varepsilon>0$ (open symbols) and $\varepsilon<0$ (full symbols) is the same (symbols are overlapping); for higher values of $n_{s}$ the volatility reaches an equilibrium level for $\varepsilon>0$ (open symbols) whilst increases for $\varepsilon<0$ (full symbols). The lowest of the two curves is the predictability $\frac{H}{P}$. For both $\varepsilon>0$ (open symbols) and $\varepsilon<0$ (full symbols) the behavior is the same (open and full symbols overlapping) and predictability goes to 0 . The different behavior of both the number of active traders $n_{a c t}$ and the volatility function $\frac{\sigma^{2}}{P}$ for $\varepsilon>0$ and $\varepsilon<0$ suggests that $\varepsilon=0$ is a point of discontinuity. 
Let us now interpret this information with the lens of minority game. For $\varepsilon>0$ (open symbols) as $n_{s}$ increases, predictability $H$ goes to 0 (lower inset, lower curve): arbitrage opportunities are exploited by speculators and the system become efficient; since arbitrage opportunities decrease, it becomes harder and harder for agents strategies to be more profitable than the threshold $\varepsilon$ (see Equation 1) and the number of active traders (upper inset, open symbols) starts decreasing helping the system to reach an equilibrium with the overall volatility $\sigma$ converging to a constant limit (lower inset, open square symbols). For $\varepsilon<0$ the behavior is completely different: since agents have a negative threshold to evaluate the goodness of their trading strategy, the number of active speculators continues increasing even if the efficient phase has been reached $(H=0$, lower inset, lower curve) and opportunities have been exploited; this imply an increase of $\sigma$ (lower inset, upper curve, full squares), and hence by Equation 2 of the market volatility $v_{m k t}$, proportional to the number of speculators $n_{s}$.

Based on these considerations, if speculators had to think in terms cost-opportunity benefit in their trading activity, the market would change from the actual $\varepsilon<0$ structure into the $\varepsilon>0$ one. Being not possible to introduce a USD mining fee (it would be in contradiction with the basic principles behind bitcoin), the introduction of future contracts on bitcoin would be equivalent to assume $\varepsilon>0$ in the GCMG. Pure financial speculators acting on the futures market would have to take into account the interest rate level in evaluating the goodness of their trading strategies: the price of a future contract includes the interest amount relative to the contract life, hence a financial speculator has to take it into account either if he is a buyer or a seller. In the first case, in order to obtain a gain, the bitcoin price appreciation has to compensate the interest amount payed, whilst in the second case the interest rate represents an income. The same concepts applies to miners: if a miner could sell bitcoins through the futures market we would be able to earn interest in USD without the constant need to try to take profit from daily price movements.

\section{Conclusions}

Bitcoin, with its clearly defined structure based on miners and long-term holders acting as producers and speculators trying to gain from market movements, offers a unique possibility to exploit capabilities of toy models, like Minority Games, to help understanding its market structure. The application of a simple model like the Grand Canonical Minority Game, able to replicate complex observable quantities of financial markets like crashes, volatility clustering and fat tail returns, suggests that the lack of a mining fee in USD for miners, that would be similar to the interest rate earned by a bond holder, could be one of the main reasons of the extremely high overall market volatility.

Coherently with the GCMG model, the introduction of future contracts on bitcoin would give miners the opportunity to earn an interest in USD and financial speculators a risk free rate to evaluate the opportunity cost of their strategies, having the effect of reducing the overall market volatility.

A possible direction of future investigation could be the application of a Minority Game model to try to answer the what is probably the most compelling question: to mine or not to mine. Even though an intuitive connection between bitcoin mining and the MG exists, an explicit mapping between the two has to be defined, especially in terms of strategies reward function. A starting point could be a paper by Challet. $^{8}$ 


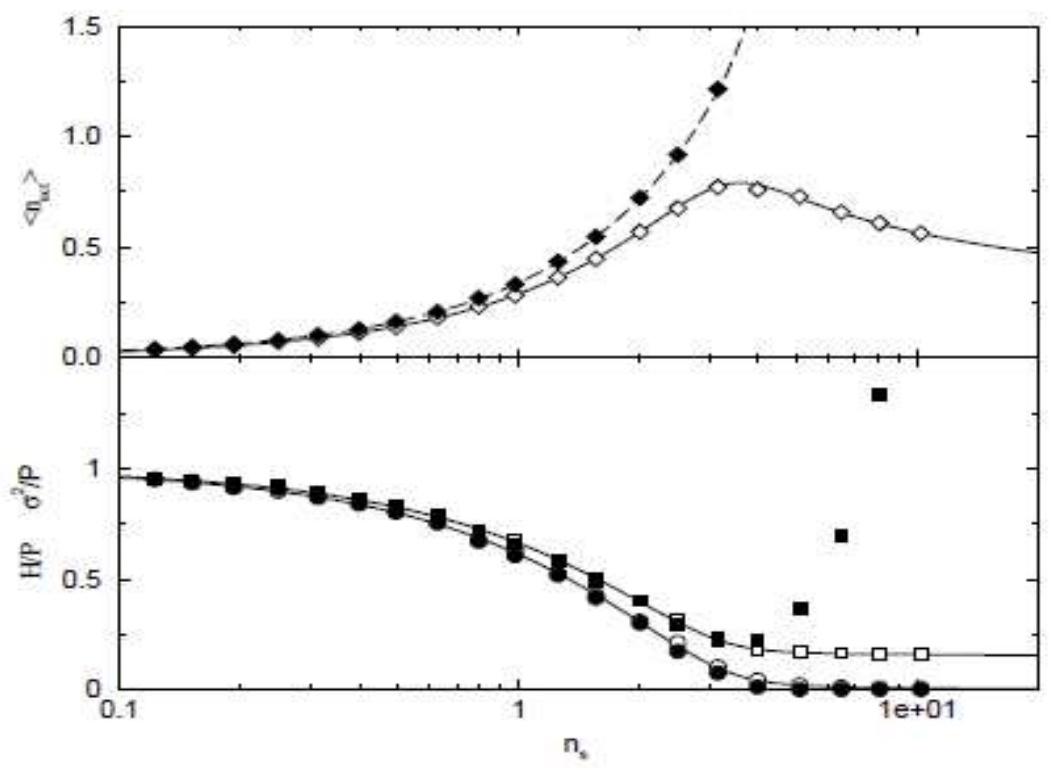

Fig. 2. Source: Challet. ${ }^{7}$ Theory and numerical simulations: number of active traders $\left\langle n_{a c t}\right\rangle$ (top), volatility $\sigma^{2} / P$ and predictability $H / P$ (bottom) as a function of number of speculators $n_{s}$ for $\varepsilon=0.1$ (solid line) and $\varepsilon=-0.01$ (dashed line). Numerical results for $\varepsilon=0.1$ (open symbols) and $\varepsilon=-0.01$ (full symbols) are averages over 200 runs.

\section{Acknowledgements}

The author wishes to thank the anonymous referees for their valuable suggestions and remarks.

\section{Notes and References}

${ }^{1}$ Nakamoto, S. "Bitcoin: A peer-to-peer electronic cash system" http://bitcoin.org/bitcoin.pdf (1994).

${ }^{2}$ Chakraborti, A., Challet, D., Chatterjee, A., Marsili, M., Zhang, Y.-C., Chakrabarti, B.K. "Statistical mechanics of competitive resource allocation using agent-based models" Physics Reports 552 1-25 (2015).

${ }^{3}$ Challet, D., Marsili, M., Zhang, Y.-C. "Stylized facts of financial markets and market crashes in Minority Games" Physica A: Statistical Mechanics and Its Applications 294.3 514-524 (2001).

${ }^{4}$ Arthur, B.W. "Inductive reasoning and bounded rationality: The El Farol problem" American Economic Review 84406 (1994).

${ }^{5}$ Black, F.S. "Noise" The Journal of Finance XLI 3 (1986).

${ }^{6}$ Moro, E. "The Minority Game: an introductory guide" http://arxiv.org/pdf/cond-mat/0402651v1.pdf (2004).

${ }^{7}$ Challet, D., Marsili, M. "Critically and finite size effects in a simple realistic model of stock market" http://arxiv.org/pdf/cond-mat/0210549.pdf (2002).

${ }^{8}$ Challet, D. "Minority mechanisms in models of agents learning collectively a resource level" Physica A: Statistical Mechanics and Its Applications 344.1 24-29 (2004).

(cc) BY

ULIS D-Sorke
Articles in this journal are licensed under a Creative Commons Attribution 4.0 License.

Ledger is published by the University Library System of the University of Pittsburgh as part of its D-Scribe Digital Publishing Program and is cosponsored by the University of Pittsburgh Press. 